\title{
ABERRANT EXPRESSION OF SELENIUM-CONTAINING GLUTATHIONE PEROXIDASES IN CLEAR CELL RENAL CELL CARCINOMAS
}

\author{
E. Rudenko ${ }^{1, *}$, O. Kondratov ${ }^{1}$, G. Gerashchenko', Y. Lapska ${ }^{1}$, S. Kravchenko', \\ O. Koliada ${ }^{2}$, S. Vozianov', Y. Zgonnyk ${ }^{3}$, V. Kashuba ${ }^{1}$ \\ ${ }^{1}$ Institute of Molecular Biology and Genetics, NAS of Ukraine, Kyiv 03680, Ukraine \\ ${ }^{2}$ Institute of Gerontology, NAMS of Ukraine, Kyiv 04114, Ukraine \\ ${ }^{3}$ Institute of Urology, NAMS of Ukraine, Kyiv 04053, Ukraine
}

\begin{abstract}
Aim: To find putative diagnostic markers for clear cell renal cell carcinomas (ccRCC). Material and methods: Quantitative polymerase chain reaction (Q-PCR), bisulfite treatment, methylation-specific PCR, analysis on cBioPortal for Cancer Genomics. Results: We have found that expression of GPX 1, GPX3, and GPX4 genes was decreased in ccRCC. We have shown that the number of alanine (GCG) repeats at the amino terminus of the GPX1 protein is variable. It was reported earlier that an allele that possess 5 alanine repeats is associated with the increased cancer risk. According to the obtained data, the allele with the 5 alanine repeats was also present in a group of healthy donors. Moreover, the frequency of alleles with repeats was similar among ceRCC patients and healthy individuals. We found that decreased expression of GPXs genes was not associated with promoter methylation. To provide other explanation, an analysis on the gene copy number was performed. We have found the heterozygous deletions for GPX1 gene, amplification for GPX3 gene, and no change in gene copy number for GPX4. Conclusions: Our data support the hypothesis that GPX1, GPX3, and GPX4 genes may play a role in ccRCC cancerogenesis and therefore they might be considered as putative diagnostic markers for ccRCC.
\end{abstract}

Key Words: clear cell renal cell carcinomas, selenium-containing GPXs, genetic and epigenetic regulation.

The clear cell renal cell carcinoma (ccRCC) represents an invasive and the most common adult kidney neoplasm. ccRCC is one of the most therapy-resistant carcinomas, responding very poorly to radio-, chemo-, and hormonal therapy [1]. So, there is an urgent need to study and understand the molecular mechanisms of ccRCC cancerogenesis with the aim to reveal the early diagnostic markers.

It is well known that cancer cells produce high amounts of reactive oxygen species that play an important role in induction of apoptosis. Cancer cells evade apoptosis, as a rule. One of mechanisms to evade apoptosis is removal of hydroperoxide. This process is under control by antioxidant enzymes that are represented by a family of glutathione peroxidases (GPXs) [2, 3].

Human GPXs family consists of 8 members; the five of them contain selenocysteine in an active center (GPX1/GPX2/GPX3/GPX4/GPX6) and other three are cysteine-containing proteins (GPX5/GPX7/GPX8). Proteins that contain selenocysteine, or selenium in the form of amino acid, are designated as selenoproteins. GPX proteins are implicated in cancerogenesis; however, the role of selenoproteins of GPX family is not fully understood. Moreover, each protein of GPX family might show different functions.

For example, gene GPX1 is localized on chromosome 3p21. This locus frequently associated with the loss of heterozygosity in cancers. The loss of heterozygosity for GPX1 was found in lung, breast, head and neck, and colon cancers [4]. Another genetic alteration is polymorphism that involves a variable

Submitted: March 13, 2015.

*Correspondence: E-mail: rudenko jene@ukr.net Abbreviations used: ccRCC - clear cell renal cell carcinoma; GPX - glutathione peroxidase; Q-PCR - quantitative polymerase chain reaction. number of "GCG" tri-nucleotide repeats in the first exon of the GPX1 gene. This results in five, six, or seven alanines near the $\mathrm{N}$-terminus. The higher number of repeats is associated with cancer of several types; it is not clear, however, which allele influences on cancer development [5].

An expression level of the GPX2 is increased in breast [6] and colorectal cancers [7, 8].

It is shown also that after transfection with a GPX3 expression construct into different prostate cancer cell lines (PC-3, DU145, and LNCaP) transfected cells exhibited decreased ability to proliferate in soft agar [9].

Expression of the GPX4 is decreased in pancreatic cancer cell lines, compared with normal human pancreas [10]. There is no data on expression pattern of the GPX6 in cancer.

The present work is devoted to a study on the role of genes encoding selenium-containing GPXs in ccRCC cancerogenesis.

\section{MATERIALS AND METHODS}

Tissue samples. All samples of tissue were obtained in accordance with the permission from the Ethical Committee of the Institute of Molecular Biology and Genetics of the NAS of Ukraine.

Surgically removed tumors and surrounding rim of normal tissues were obtained from Kyiv National Urological Center (Ukraine). All tumor samples were characterized, according to the International System of Clinico-Morphological Classification of Tumors (TNM), based on the tumor-node-metastasis and staging classification (1989) [11] and WHO criteria classification (1999) [12]. The mean age of patients was $56 \pm 3.75$ years, with a male to female ratio of $58: 42$, respectively. The samples were grouped, 
according to the stage of tumor development and arranged in order of increasing atypia.

DNA of healthy donors was received from Institute of Gerontology of the NAMS of Ukraine (License of the Ministry of Health AB 511829). The mean age of patients was $65 \pm 4.5$ years, with a male to female ratio of $1: 1$.

Isolation of genomic DNA and total RNA. Genomic DNA was isolated, using the DNA purification Kit (Fermentas, Lithuania), according to the manufacturer's recommendations. Total RNA was extracted with the RNeasy Mini Kit (QIAGEN, USA), following the producer's protocol. Quality of genomic DNA and total RNA was assessed by agarose gel electrophoresis; their concentration was measured, using a spectrophotometer Nano Drop ND-1000 (NanoDrop Technologies Inc., USA). The samples, used for the quantitative polymerase chain reaction (Q-PCR), were of high molecular weight and pure from contaminations (an OD 260/280 ranging from 1.6 to 1.8). From each RNA sample, $1 \mu \mathrm{g}$ of total RNA was treated with DNAse I and reversely transcribed in duplicates, as well as negative controls without enzyme, using the RevertAid ${ }^{\mathrm{TM}} \mathrm{H}$ Minus First Strand cDNA Synthesis Kit (Thermo Scientific, SE, Sweden).

Analysis of gene expression levels. The analysis of relative expression of genes was performed for GPX1 and GPX3 on 12 cCRCC samples and corresponding normal tissues, and for 16 paired samples for GPX-2, -4, and - 6 by Q-PCR, using the IQ5 Cycler (Bio Rad, USA) and Mastermix SYBR Green (Thermo Scientific, SE, Sweden) according to the manufacturer's instruction. Primers for each gene (Table 1) were designed, using the Primer3 program (http://frodo.wi.mit.edu/primer3/). Each Q-PCR reaction mix contained $12.5 \mu \mathrm{l}$ of $2 \times$ SYBR Green PCR Master Mix (Fermentas, USA), $0.3 \mathrm{mM}$ of primers, $10 \mathrm{ng} / \mu \mathrm{l} \mathrm{cDNA}$, and a nuclease-free water to make a total volume of $25 \mu \mathrm{l}$. TBP was used as reference gene [13]. The change of expression was calculated with $\Delta \Delta$ Ct method of relative quantification [14].

Table 1. Primers for target and reference genes to study expression levels

\begin{tabular}{lcc}
\hline Gene & Forward primer & Reverse primer \\
\hline GPX1 & CCAAGCTCATCACCTGGTCT & TCGATGTCAATGGTCTGGAA \\
GPX2 & TTTTGGACAAGGGTGAAGGT & TGCAACCAATTTGGACATCA \\
GPX3 & TACGAGTACGGAGCCCTCAC & CCAGAATGACCAGACCGAAT \\
GPX4 & ATGTCCTTGGCGGAAACTC & CCTTGGGTGGATCTTCATC \\
GPX6 & AGGGTGAGGGCTCCATACTC & AGTTCCAGGCCTCCTGTCTT \\
TBP & GAACCACGGCACTGATTTC & CACAGCTCCCCACCATATTC \\
PCR conditions: $50^{\circ} \mathrm{C} \times 2$ min, $95^{\circ} \mathrm{C} \times 10$ min, 35 cycles of $95^{\circ} \mathrm{C} \times 20 \mathrm{~s}$, \\
$60^{\circ} \mathrm{C} \times 20 \mathrm{~s} 72^{\circ} \mathrm{C} \times 40 \mathrm{~s}$. & \\
\hline
\end{tabular}

Analysis of trinucleotide repeat variations. Analysis of trinucleotide repeat variations was performed on $40 \mathrm{ccRCC}$ and 41 normal tissues. The reaction conditions and sequence of primers are shown in Table 2. Forward primer was labelled by Cy5dye (IBA, USA).

Table 2. Primers for GPX1 to study the GCG repeat polymorphism (number of Ala repeats)

\begin{tabular}{clc}
\hline Gene & \multicolumn{1}{c}{ Forward primer } & \multicolumn{1}{c}{ Reverse primer } \\
\hline GPX1 & Cy5-GAAAACTGCCTCTGC- & TGCAACCAATTTGGACATCA \\
& CACGTGACC &
\end{tabular}

PCR conditions: $94^{\circ} \mathrm{C} \times 2 \mathrm{~min}, 28$ cycles of $94^{\circ} \mathrm{C} \times 30 \mathrm{~s}, 68^{\circ} \mathrm{C} \times 20 \mathrm{~s} 72^{\circ} \mathrm{C} \times 60 \mathrm{~s}$.
Denaturing PAAG electrophoresis was used to determine the exact size of trinucleotide repeats. The electrophoresis was conducted on automatic laser sequencing machine "ALF express" (Pharmacia Biotech, USA). The mixture that contained $3 \mu$ of amplification product and $2 \mu \mathrm{l}$ of loading buffer $(0.01 \%$ bromphenol blue, $0.01 \%$ xylene cyanol, $98 \%$ formamide), was denatured at $95^{\circ} \mathrm{C}$ for 5 min before electrophoresis.

Identification of $\mathrm{CpG}$ islands in the promoter region of GPX genes. The $\mathrm{CpG}$ islands in the promoter region of GPXs were defined, using $\mathrm{CpG}$ island searcher online tool (http://www.uscnorris.com/ cpgislands2/cpg.aspx).

DNA bisulfite treatment and methylationspecific PCR (MSP). Bisulfite modification of the purified DNA from 25 ccRCC samples along with 25 corresponding normal tissues was achieved, using the EZ DNA Methylation Kit (Zymo Research Corporation, USA), following the producer's protocol. Methyl specific Q-PCR was done. Each Q-PCR reaction mix contained $12.5 \mu \mathrm{l}$ of $2 \times$ SYBR Green PCR Master Mix (Thermo Scientific, SE, Sweden), $0.3 \mathrm{mM}$ of primer, $10 \mathrm{ng} / \mathrm{\mu l}$ of genomic DNA, and a nuclease-free water to make a total volume of $25 \mu \mathrm{l}$. Q-PCR was carried out for 35 cycles comprising $15 \mathrm{~s}$ denaturation at $95^{\circ} \mathrm{C}$ and $60 \mathrm{~s}$ annealing at $60^{\circ} \mathrm{C}$. The cycling was started by $50^{\circ} \mathrm{C} \times 2 \mathrm{~min}, 95^{\circ} \mathrm{C} \times 10 \mathrm{~min}$. Primers for MSP (Table 3 ) targeting CpG-rich promoter region were designed, using online software "MethPrimer" (http://www.urogene.org/methprimer/). COL2A1- was used as reference gene.

Table 3. Primers for target and reference genes to study deletion search and methylation analysis

\begin{tabular}{lll}
\hline \multicolumn{1}{c}{ Gene } & \multicolumn{1}{c}{ Forward primer } & \multicolumn{1}{c}{ Reverse primer } \\
\hline GPX1M & CTAACCGACAACACACATAACG & GAGGCGGGATTTAGGTC \\
GPX1unM ACCAAACAACACACATAACACA & ATGAGGTGGGATTTIAGGTTT \\
GPX3M & GTTGAGGGAAGTCGCGTTC & GTCCGTCTAAATATCCGACG \\
GPX3unM & GAGTTGAGGGTAAGTTGTGTTGT & CCATCCATCTAAAATATCCAA- \\
& & CACT \\
GPX4M & GTTGGAAATTTCGGATTACGC & ACCGTAATCAAAACTACGCG \\
GPX4unM & GGGTGGAAATTTGGATTATGT & CCAAAACCATAATCAAAAC- \\
& & TACAA \\
COL2A1 & GTAATGTTAGGAGTATTITGTGGGA CTACCCCAAAAAACCCAATCCTA \\
\hline
\end{tabular}

Analysis of gene copy number variation. Analysis of variation of gene copy number was performed on 12 ccRCC samples, in parallel with normal tissues from the same patients, as described earlier [14].

Analysis of the open-access bio-database. The cBioPortal for Cancer Genomics provides visualization, analysis and download of large-scale cancer genomics data sets (http://cbioportal.org). Analysis of 415 ccRCC samples in comparison with corresponding normal tissues was performed in silico.

Statistical analysis. Allele frequency and genotype analysis was performed, using the Hardy Weinberg equilibrium with Genepop v4 software (http://genepop.curtin.edu.au/) [15]. The Nonparametric Wilcoxon test was used to compare mRNA expression of target and reference genes for the same sample. Groups of samples were compared in respect to average level of mRNA decrease (LDav) and the frequency of decrease (FD). The LD was calcu- 
lated as $1 / R$ and reflects the $n$-fold factor by which the mRNA content decreased in the tumor compared to normal tissue. Nonparametric Kruskal - Wallis and Mann - Whitney rank-sum tests were used to test mRNA differences (both LDav and FD) for each target gene. The Nonparametric Spearmen's criterion was used to calculate the coefficient of correlation between the LDav for each set of pairs of target genes. P-values $<0.05$ were considered statistically significant. All statistical procedures were performed using the BioStat software.

\section{RESULTS AND DISCUSSION}

Expression of the GPX family genes in $\boldsymbol{C C R C C}$. We have studied expression of all five selenium-containing GPXs.

GPX1. We have shown that GPX1 expression was decreased in $85 \%$ (9 of 12 ) of ccRCC cases.

Protein, encoded by GPX1 that is located on chromosome 3p21.3, was found in the cytosol and mitochondria. The primary function of GPX1 protein is detoxifying of hydroperoxides. GPX1 cannot be replaced by any other selenoprotein in protecting from generalized oxidative stress. GPX1 can prevent DNA mutations due to oxidative stressand also counteracts production of COX/LOX-derived pro-inflammatory mediators. Thereby, GPX1 might prevent cancerogenesis, at least at the initiation stage [16]. Few studies have shown protective effect of GPX1; GPX1 overexpression resulted in reduced proliferation of tumor cell lines in vitro and in experimental animals (xenograft model) [17].

GPX1 is polymorphic at several positions and some alleles have been associated with the increased cancer risk. The most studied GPX1 polymorphism is proline/ leucine variation at codon 198. Another example of polymorphism is the variable number of alanine (GCG) repeats at the amino terminus of the protein. It was shown that there are three different types of alleles, with five, six or seven (Ala) repeats in this sequence. It was also reported that there is an association of allele with 5 alanine repeats and the increased the risk of prostate cancer in young patients [18]. So, we wanted to investigate the relationship between the GCG repeat polymorphism in GPX1 sequence and ccRCC cancerogenesis.

An analysis of the GCG repeat polymorphism (number of Ala repeats) in GPX1 sequence was performed and genotypes distribution was studied in ccRCC patients and in the control group (healthy individuals) (Table 4). No deviations from Hardy - Weinberg equilibrium was found in the both groups. Five Ala repeats was found in $39(48.8 \pm 5.6 \%)$, six repeats in $24(30.0 \pm$ $5.1 \%)$, and seven - in $17(21.2 \pm 4.6 \%)$ of ccRCC patients. In the control group Ala repeats were distributed similarly: five repeats was found in $39(47.6 \pm 5.5 \%)$, six - in $22(26.8 \pm 4.9 \%)$, and seven - in $21(25.6 \pm$ $4.8 \%$ ) of healthy donors. By other words, no significant difference was observed between ccRCC patients and the control group.

As is seen from Table 4, five Ala repeats was detected the most frequently in the both groups. An obvi- ous question was to check whether the five Ala repeats would be found at a higher rate among ccRCC patients in comparison with healthy persons. No difference was found, however, between patients and healthy individuals $(p=0.944)$.

Table 4. Distribution of GPX1 genotype for ccRCC patients and healthy individuals

\begin{tabular}{lcc}
\hline $\begin{array}{c}\text { GPX1 (№ of Ala repeats } \\
\text { on both alleles) }\end{array}$ & $\begin{array}{c}\text { Renal cancer, } \\
\mathrm{n}(\%)(\mathrm{n}=40)\end{array}$ & $\begin{array}{c}\text { Control, } \mathrm{n}(\%) \\
(\mathrm{n}=41)\end{array}$ \\
\hline $5 / 5$ & $10(25.0 \pm 6.8)$ & $11(26.8 \pm 6.9)$ \\
$6 / 6$ & $4(10.0 \pm 4.7)$ & $2(4.9 \pm 3.4)$ \\
$7 / 7$ & $1(2.5 \pm 2.5)$ & $4(9.8 \pm 4.6)$ \\
$5 / 6$ & $10(25.0 \pm 6.8)$ & $11(26.8 \pm 6.9)$ \\
$5 / 7$ & $9(22.5 \pm 6.6)$ & $6(14.6 \pm 5.5)$ \\
$6 / 7$ & $6(15.0 \pm 5.6)$ & $7(17.1 \pm 5.9)$ \\
Heterozygote frequencies & $25(62.5 \pm 7.7)$ & $24(58.5 \pm 7.7)$ \\
Homozygote frequencies & $15(37.5 \pm 7.7)$ & $17(41.5 \pm 7.7)$ \\
\hline
\end{tabular}

GPX2. GPX2 is located on chromosome 14q24.1 and shows upregulated expression in colorectal cancer, Barrett's esophagus, squamous cell carcinoma and lung adenocarcinoma of smokers. From other hand, it was shown that GPX2 might prevent tumorigenesis associated with inflammation. Hence, this gene could show different properties upon cell transformation, compared with normal cells. It was proposed that GPX2 may prevent initiation but support promotion of cancerogenesis [19]. This hypothesis is supported by the fact that GPX2 expression is only transiently upregulated in early stages of malignant transformation in the colon [20].

Noteworthy, no expression of GPX2 gene in the normal and tumor tissues of kidney was registered in the present work.

GPX3. We have shown that expression of GPX3 (located on chromosome 5q33.1) was decreased in cCRCC in $100 \%$ of cases. Inhibition of expression of GPX3 and as consequence, activity of GPX3 protein, might be associated with different stages of cancer progression, including initiation, promotion, and metastasizing [21]. Moreover, GPX3 expression was downregulated in the plasma of patients bearing breast, gastric, and colorectal cancers. In cancer tissues of prostate, esophagus, and thyroid GPX3 expression was decreased as well $[22,16]$.

GPX4. Expression of GPX4 (located on chromosome 19p13.3) at the mRNA level was decreased in $81 \%(13 / 16)$ of samples, when tumors were compared with corresponding normal tissues.

GPX4 is an ubiquitously expressed anti-oxidant protein that, in contrast to other GPX isoforms, resides in the cell membrane and can reduce and detoxify lipid hydroperoxides [23]. GPX4 exists in three different isoform, i.e. cytoplasmic, mitochondrial, and sperm nuclear. GPX4 plays an important role in cell functioning, because GPX4 knockout is embryonically lethal [24].

GPX6. GPX6 (located on chromosome 6p22.1) is restricted in expression to the developing embryo and olfactory epithelium in adults. This protein has not been yet characterized in terms of function or disease [25]. No expression of GPX6 was found in the normal and cancer kidney tissues in our study. 
Methylation profile of GPXs promoter region in ccRCC tissue samples. The $\mathrm{CpG}$ islands in the promoter region of GPXs were defined, using $\mathrm{CpG}$ island searcher online tool (http://www.uscnorris.com/cpgislands2/cpg.aspx). We have found $\mathrm{CpG}$ islands in promoter region of GPX1, GPX3, and GPX4, but not of GPX2 and GPX6 genes. 25 ccRCC samples and respective normal tissues were analyzed, using the methyl-specific quantitative PCR (MSP qPCR) and primers for unmethylated and methylated forms of genes. The obtained results showed that there was no methylation in any tumor samples. Hence, decreased expression of GPXs genes is not associated with promoter methylation.

Analysis of variations in GPXs copy number. Analysis of GPXs gene copy number was performed on 12 renal cancer samples. Changes of GPX1 gene copy number were observed in 92\% (11/12) of RCC samples; 11 samples displayed ratio $<0.7$ and $>0.2$ and that was considered as heterozygous deletions. One sample showed ratio 1.2 and that was considered as unchanged status. Changes of GPX3 gene copy number were observed in 50\% (6/12) of RCC samples. The ratio was $>1.4$ and that was considered as amplification. Copy number of GPX4 was not changed (Table 5).

Table 5. Summary on GPXS copy number in the tumor tissues

\begin{tabular}{ccccccc}
\hline $\begin{array}{c}\text { Sample } \\
\text { number }\end{array}$ & TNM & $\begin{array}{c}\text { Stage } \\
\text { of atypia }\end{array}$ & Grade & $\begin{array}{c}\text { Ratio T/N } \\
\text { GPX1 }\end{array}$ & $\begin{array}{c}\text { Ratio T/N } \\
\text { GPX3 }\end{array}$ & $\begin{array}{c}\text { Ratio T/N } \\
\text { GPX4 }\end{array}$ \\
\hline 1 & T4NxM0 & 3 & IV & 1.2 & 1.1 & 1.1 \\
2 & T2aNxM1 & 3 & IV & 0.4 & 1.4 & 1.1 \\
3 & T2NxM1 & 4 & IV & 0.2 & 2.2 & 1.1 \\
4 & T2aNxM1 & 3 & II & 0.7 & 1.1 & 1.2 \\
5 & T4NxM0 & 4 & IV & 0.3 & 0.8 & 0.9 \\
6 & T4N0M1 & 3 & IV & 0.4 & 1.5 & 1.1 \\
7 & T3bNxM0 & 2 & III & 0.7 & 0.9 & 1.1 \\
8 & T3bNxM0 & 2 & III & 0.7 & 0.9 & 1.0 \\
9 & T1bNxM0 & 2 & II & 0.4 & 1.0 & 0.9 \\
10 & T3N1M0 & 3 & III & 0.4 & 1.4 & 0.9 \\
11 & T3N0M0 & 3 & III & 0.3 & 1.5 & 1.0 \\
12 & T3N0M0 & 3 & III & 0.6 & 1.5 & 1.0 \\
\hline
\end{tabular}

All the data discussed above suggest that changes (decrease) of expression of GPXs genes are achieved by the different mechanisms upon cancerogenesis.

To validate our data, the bioinformatic analysis was performed, using cBioPortal Web resource online [26, 27]. The cBioPortal for Cancer Genomics allow us exploring, visualizing, and analysis of multidimensional cancer genomics data. This portal compress the data on molecular profiling of different cancer tissues and also different data bases (for example microarrays, RNAseq, etc.). Large-scale cancer genomics projects, such as The Cancer Genome Atlas (TCGA) and the International Cancer Genome Consortium (ICGC) generate an overwhelming amount of cancer genomics data from the multiple different technical platforms, making it increasingly challenging to perform data integration, exploration, and analysis. Genetic alterations in 415 ccRCC were analyzed, using cBioPortal Web. The obtained results are shown on Figure.
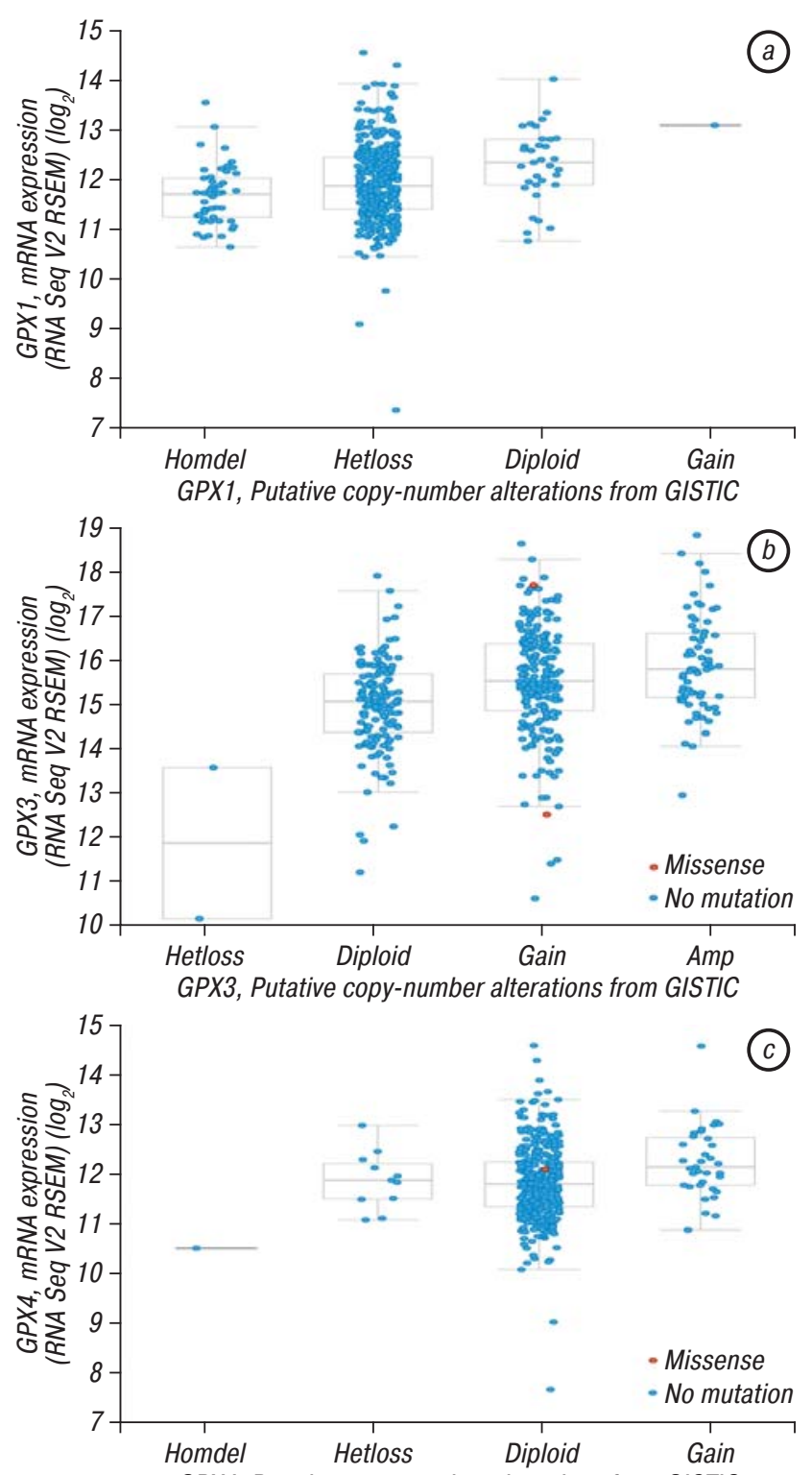

GPX4, Putative copy-number alterations from GISTIC

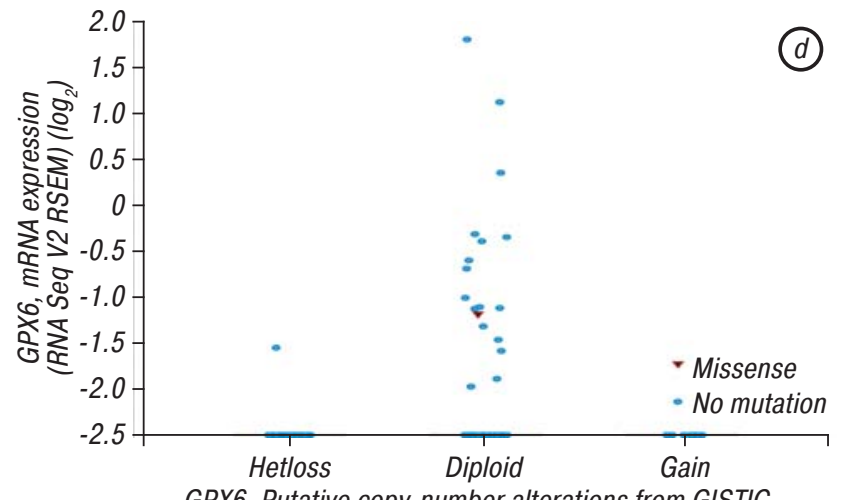

GPX6, Putative copy-number alterations from GISTIC

Figure. Relative expression level as a function of relative copy number of GPXs genes. Homdel — homozygously deleted; Hetloss - heterozygously deleted; Diploid - two alleles present; Gain - low-level gene amplification event; Amp — high-level gene amplification event

As seen on Figure, our results are in line with the data obtained by other methods. So, gene GPX1 is heterozygously deleted, gene GPX3 showed the low level of gene amplification event, and for GPX4 no changes were observed. As was mentioned before, genes GPX2 and GPX6 were not expressed in ccRCC tumors nor in normal tissues. 
The main reasons of decreased gene expression are deletion, promoter methylation, and downregulation by proteins or miRNAs. In case of ccRCC, methylation is not a cause of decreased expression of GPXs. We have to mention, that decreased expression of gluthation peroxidases can be both, the cause and the consequence of cancerogenesis; their transcription is regulated by glutathione oxidative potential, which is low in tumors [28]. Such regulation is provided by several mechanisms that are common for all GPXs. First, there are oxygen response elements, ORE for activation or inhibition of transcription by "proteins-sensors" in GPX promoter sequence. Interestingly, hyperoxia increases transcription of GPX 1 in endothelial cells of umbilical vein through a mechanism independent of ORE, suggesting that transcription of GPXs in response to oxygen stress is regulated by more than one mechanism [29, 30]. Second, oxidative potential in cell impacts on the catalytic activity of GPXs by regulating the availability of protein SBP2, which is required for the insertion of selenium [4]. Hence, the role of GPXs in cancerogenesis cannot be considered only as the antioxidant proteins, as was mentioned above. It depends also on types and stage of cancer and class of GPXs [16].

Downregulation of expression of GPXs genes is observed at the early stages of ccRCC [31], so it can be one of the causes of cancer initiation. Expression is decreased even more upon the cancerogenesis, and it may be explained by two assumptions. Firstly, it may reduce the oxidative capacity of the cells, due to the fact that cancer cells are more exposed to hypoxia upon increase of tumor size. Secondly, the p53 protein can bind to the promoter of the gene as a transcription factor, thus, stimulating transcription [32, 33]. So, reduced expression of GPX may be explained by inactivation of p53 function, which is typical for majority of tumors.

\section{CONCLUSIONS}

In the present work we have found that GPX1, GPX3, and GPX4 genes showed decreased expression in tumors, compared with the normal tissues. GPX2 and GPX6 genes were not expressed in renal cell carcinomas cancer or normal tissues. Promoters of the studied genes were not methylated in renal cell carcinomas cancer samples. So, lowered expression of GPX genes is not associated with promoter methylation. Other mechanism must be responsible for decrease of GPX expression. We have found heterozygous deletions in GPX1 gene. GPX3 gene, in contrast, showed amplification; no changes in copy number of GPX4 gene was found. Despite the uncertainty what was a cause of decreased expression of genes (except GPX1), GPXs might be considered as diagnostic markers for ccRCC at the certain circumstances.

\section{REFERENCES}

1. Tannir NM. Renal cell carcinoma. Oxford: Oxford University Press, 2014.

2. Nogueira V, Hay N. Molecular pathways: reactive oxygen species homeostasis in cancer cells and implications for cancer therapy. Clinical Cancer Res 2013; 19: 4309-14.
3. Brigelius-Flohé R, Maiorino M. Glutathione peroxidases. Biochim Biophys Acta 2013; 1830: 3289-303.

4. Lubos E, Loscalzo J, Handy D. Glutathione peroxidase-1 in health and disease: from molecular mechanisms to therapeutic opportunities. Antioxid Redox Signal 2011; 15: 1957-97.

5. Zhuo P, Goldberg M, Herman L, et al. Molecular consequences of genetic variations in the glutathione peroxidase 1 selenoenzyme. Cancer Res 2009; 69: 8183-90.

6. Naiki-Ito A, Asamoto M, Hokaiwado $\mathrm{N}$, et al. $\mathrm{Gpx} 2$ is an overexpressed gene in rat breast cancers induced by three different chemical carcinogens. Cancer Res 2007; 67: 11353-8.

7. Murawaki Y, Tsuchiya H, Kanbe T, et al. Aberrant expression of selenoproteins in the progression of colorectal cancer. Cancer Lett 2008; 259: 218-30.

8. Lin Y, Furukawa Y, Tsunoda T, et al. Molecular diagnosis of colorectal tumors by expression profiles of 50 genes expressed differentially in adenomas and carcinomas. Oncogene 2002; 21: 4120-8.

9. Yu Y, Yu G, Tseng G, et al. Glutathione peroxidase 3, deleted or methylated in prostate cancer, suppresses prostate cancer growth and metastasis. Cancer Res 2007; 67: 8043-50.

10. Liu J, Du J, Zhang Y, et al. Suppression of the malignant phenotype in pancreatic cancer by overexpression of phospholipid hydroperoxide glutathione peroxidase. Hum Gene Ther 2006; 17: 105-16.

11. Speissel B, Beahrs O, Hermanek P, et al. TNM atlas: illustrated guide to the TNM/pTNM classification of malignant tumours Berlin. New York: Springer, 1989. 343 p.

12. Travis W, Coby T, Corrin B, et al. World Health Organization International histological classification of tumours; histological typing of lung and pleural tumours. Berlin: Springer, 1999. $156 \mathrm{p}$.

13. Jung M, Ramankulov A, Roigas J, et al. In search of suitable reference genes for gene expression studies of human renal cell carcinoma by real-time PCR. BMC Mol Biol 2007; 8: 47-9.

14. Gerashchenko G, Bogatyrova $O$, Rudenko E, et al. Genetic and epigenetic changes of NKIRAS1 gene in human renal cell carcinomas. Exp Oncol 2010; 32: 71-5.

15. Rousset F. Genepop'007: a complete reimplementation of the Genepop software for exact tests for Windows and Linux. Mol Ecol Resour 2008; 8: 103-6.

16. Brigelius-Flohé R, Kipp A, Liu J, et al. Glutathione peroxidases in different stages of carcinogenesis. In: Human Gene Therapy, 2004: 239-50.

17. Zhuo P, Diamond A. Molecular mechanisms by which selenoproteins affect cancer risk and progression. Biochim Biophys Acta 2009; 1790: 1546-54.

18. Kote-Jarai Z, Durocher F, Edwards SM, et al. Association between the GCG polymorphism of the selenium dependent GPX1 gene and the risk of young onset prostate cancer. Prostate Cancer Prostatic Diseases 2002; 5: 189-92.

19. Brigelius-Flohé R, Kipp AP. Physiological functions of GPX2 and its role in inflammation-triggered carcinogenesis. Ann NY Acad Sci 2012; 1259: 19-25.

20. Florian S, Wingler K, Schmehl K, et al. Cellular and subcellular localization of gastrointestinal glutathione peroxidase in normal and malignant human intestinal tissue. Free Radic Res 2001; 35: 655-63.

21. Mohamed M, Sabet S, Peng DF, et al. Promoter hypermethylation and suppression of glutathione peroxidase 3 are associated with inflammatory breast carcinogenesis. Oxidative Medicine and Cellular Longevity 2014; 2014 (http://dx.doi.org/10.1155/2014/787195).

22. Pawlowicz Z, Zachara B, Trafikowska U, et al. Blood selenium concentrations and glutathione peroxidase activities 
in patients with breast cancer and with advanced gastrointestinal cancer. J Trace Elem Electrolytes Health Dis 1991; 5: 275-7.

23. Cole-Ezea P, Swan D, Shanley D, et al. Glutathione peroxidase 4 has a major role in protecting mitochondria from oxidative damage and maintaining oxidative phosphorylation complexes in gut epithelial cells. Free Radical Biol Med 2012; 53: 488-97.

24. Imai H, Hirao F, Sakamoto T, et al. Early embryonic lethality caused by targeted disruption of the mouse PHGPX gene. Biochem Biophys Res Commun 2003; 305: 278-86.

25. Kryukov G, Castellano S, Novoselov S, et al. Characterization of mammalian selenoproteomes. Science 2003; 300: 1439-43.

26. Cerami E, Gao J, Dogrusoz U, et al. The cBio cancer genomics portal: an open platform for exploring multidimensional cancer genomics data. Cancer Discov 2012; 2: 401-4.

27. Gao J, Aksoy BA, Dogrusoz G, et al. Integrative analysis of complex cancer genomics and clinical profiles using the cBioPortal. Sci Signal 2013; 6: pl1.
28. Koch C, Evans S. Optimizing hypoxia detection and treatment strategies. Semin Nucl Med 2015; 45: 163-76.

29. Merante F, Altamentova S, Mickle D, et al. The characterization and purification of a human transcription factor modulating the glutathione peroxidase gene in response to oxygen tension. Mol Cell Biochem 2002; 229: 73-83.

30. Jornot L, Junod A. Hyperoxia, unlike phorbol ester, induces glutathione peroxidase through a protein kinase C-independent mechanism. Biochem J 1997; 326: 117-23.

31. Rudenko E, Gerashchenko G, Lapska Y, et al. Genetic and epigenetic changes of GPX1 and GPX3 in human clearcell renal cell carcinoma. Biopolym Cell 2013; 29: 395-401.

32. Sablina A, Budanov A, Ilyinskaya $G$, et al. The antioxidant function of the p53 tumor suppressor. Nat Med 2005; 11: $1306-13$.

33. Tan M, Li S, Swaroop M, et al. Transcriptional activation of the human glutathione peroxidase promoter by $\mathrm{p} 53$. Biol Chem 1999; 274: 12061-6. 